\title{
Vantaggi clinici nella valutazione dell'urea. Ruolo del monitoraggio mediante biosensore
}

\author{
G. Bazzato, F. Saccoman \\ Divisione di Nefrologia e Dialisi, Ospedale Umberto I - Mestre Venezia
}

ecenti studi americani hanno dimostrato che la morbilità e la mortalità dei pazienti in trattamento dialitico cronico è correlata significativamente alla dose dialitica erogata.

Inoltre è stato osservato che le basse dosi di dialisi prescritte, causavano una elevata ospedalizzazione di questi pazienti.

Tuttavia questi dati sono stati desunti da studi retrospettivi dai quali appariva che il tempo di trattamento dialitico e la caduta intradialitica dell'urea, indice della dose dialitica, erano in relazione inversa con l'incremento della mortalità.

Parimenti è stato evidenziato che minore ospedalizzazione e maggiore sopravvivenza era presente nei pazienti che ricevevano elevata dose di dialisi.

Sulla base di queste osservazioni cliniche è scaturita la necessità di valutare la dose di dialisi in rapporto alla morbilità e mortalità che questa comporta.

Pertanto la quantificazione della terapia è ora considerata uno standard-care per assicurare un livello accettabile di terapia dialitica; ciò è stato dimostrato chiaramente dalla relazione di dose dialitica misurata da $\mathrm{Kt} / \mathrm{V}$ o indice di riduzione dell'urea con la morbilità e mortalità dei pazienti in emodialisi, come riferito da Clark et al (1).

Sebbene ci sia univocità di vedute nel ritenere necessario quantificare la dose di dialisi, non esiste però un metodo specifico che fornisca dati attendibili.

Sargent e Gotch (2) hanno descritto un modello di single-pool a volume variabile dell'Urea Kinetic Modeling (UKM) impiegato da circa 20 anni negli Stati Uniti, senza però trovare diffusa applicazione per la sua complessità matematica.

Successivamente altre tecniche più semplici, sono state adottate in studi clinici per dimostrare la correlazione tra dose dialitica e condizioni generali dei pazienti.

\section{Come può essere definita la dose dialitica adeguata da prescrivere?}

Secondo De Palma per dose dialitica adeguata (D.D.A.) si intende un trattamento che permetta al paziente di riabilitarsi, nutrirsi correttamente, stimolare l'apparato emopoietico, mantenere livelli pressori del sangue normali e prevenire la comparsa di neuropatie.

A questi dati clinici corrispondono le caratteristiche del trattamento dialitico quali: biocompatibilità, correzione dell'equilibrio acido-base, rimozione dei fluidi, omeostasi emodinamica e buona efficienza depurativa.

Per soddisfare questi requisiti il National Cooperative Dialysis Study (NCDS) dimostrò che i pazienti con maggiore concentrazione di urea, presentavano più complicanze rispetto a quelli a più bassi livelli, confermando la correlazione tra incidenza di problematiche cliniche e concentrazione di urea.

Pertanto anche se non è stata individuata una singola sostanza responsabile della sindrome uremica, è chiaro che l'urea rappresenta il prodotto finale del metabolismo proteico ed è la più comune delle piccole molecole; non è di per sé tossica ma è correlata con la tossicità. È un marker ideale per altre sostanze tossiche che diffondono analogamente all'urea attraverso la membrana di dialisi; inoltre è il più importante indice che fornisce informazioni sul metabolismo proteico.

Si è individuata perciò l'urea quale marker ideale in quanto la sua cinetica dà informazioni sul trattamento e la dieta del paziente.

In particolare $\mathrm{i}$ dati ottenuti dalla cinetica dell'urea sono di due tipi: 1) sul paziente quali il volume di distribuzione dell'urea (V), generazione di urea $(\mathrm{G})$ e la velocità di catabolismo proteico (PRC), 2) sulla dialisi quali concentrazione di urea (TAC), clearance del dializzatore $(\mathrm{K})$, indice di dose dialitica $(\mathrm{Kt} / \mathrm{V})$, tempo di dialisi ideale $(\mathrm{t})$. 


\section{Come si misura la dose di dialisi?}

La dose dialitica è misurata dall'indice $\mathrm{Kt} / \mathrm{V}$ definita da Gotch (2) come clearance frazionale del volume di distribuzione di un soluto dove: $\mathrm{K}$ = clearance del dializzatore, $\mathrm{t}=$ tempo di seduta di dialisi, $\mathrm{V}=$ volume di distribuzione dell'urea.

Per calcolare il $\mathrm{Kt} / \mathrm{V}$ si può moltiplicare la $\mathrm{K}$ stimata per il tempo e dividere per il volume di distribuzione, oppure si può calcolare con modelli cinetici che permettono di legare la concentrazione dell'urea di un dato istante della dialisi $(\mathrm{Ct})$ alla concentrazione di urea predialitica (Co).

Questi ultimi modelli consentono di riprodurre la realtà e per quanto attiene la cinetica dell'urea sono modelli matematici che mirano a descrivere in tempo reale la concentrazione dell'urea durante la dialisi e tra una dialisi e l'altra.

La cinetica classica dell'urea si basa sul metodo dei prelievi di sangue che comportano però molteplici errori metodologici legati a questa tecnica.

L'alternativa è rappresentata dal monitoraggio della cinetica dell'urea misurata nel sangue intero, nel plasma, nell'acqua plasmatica, nelle urine e in altri fluidi corporei, valutandola in continuo nel paziente durante la dialisi con l'impiego di sistemi integrati interfacciati con sensori specifici dell'urea.

Le tecniche più comuni utilizzano l'enzima ureasi responsabile dell'idrolisi dell'urea secondo la relazione: "Urea $+2 \mathrm{H}_{2} \mathrm{O}$ $<<<$ ureasi $>>>$ ammonio + bicarbonato". Gli ioni ammonio liberati dalla relazione in quantità proporzionale alla concentrazione di urea, vengono rilevati da reazioni colorimetriche, misura di conducibilità, $\mathrm{pH}$ e ioni selettivi.

I metodi finora applicati in dialisi sono di 2 tipi: dosaggio nel liquido di dialisi e nell'acqua plasmatica, compatibilmente con la tecnologia disponibile, utilizzando dei biosensori.

Il biosensore è un dispositivo in grado di

TABELLA I - DOSE DI DIALISI: Kt/V

\begin{tabular}{lcc}
\hline Prescritta & Calcolata & Somministrata \\
\hline $1.56 \pm 0.28$ & $1.50 \pm 0.28$ & $1.37 \pm 0.23$ \\
\hline
\end{tabular}

misurare la concentrazione di un soluto specifico in un ambiente biologico.

E costituito da un substrato + enzima e da un trasduttore.

L'elemento biologico è la parte sensibile del biosensore (ureasi) che reagisce con la sostanza bersaglio (urea) fornendo un segnale al trasduttore che lo converte in entità elettrica facilmente misurabile.

L'approccio per il monitoraggio on-line dell'urea proposto dalla ditta Bellco (3), si svolge nell'acqua plasmatica.

La tecnica dialitica PFD integrata dal monitoraggio on-line dell'urea, permette di valutare l'efficienza del trattamento stesso offrendo al medico gli strumenti di indagine alla conoscenza delle condizioni cliniche del paziente.

L'importanza dell'impiego delle metodiche sopraccennate trova conferma nei molteplici contributi di studio forniti da gruppi americani i quali hanno dimostrato come l'aumento di dose dialitica abbia ridotto la morbilità dei pazienti, dimostrando altresì la differenza tra la dose prescritta, calcolata e somministrata ai vari pazienti (Tab. I).

Merita di essere segnalato lo studio sugli effetti della dose di trattamento sulla morbilità e sulla mortalità dei pazienti in dialisi, condotto da Hakim et al (4).

Questi Autori hanno dimostrato chiaramente come l'aumento dei tempi medi di dialisi da 195 a $212 \mathrm{~min}$. e la clearance dell'urea da 170 a $220 \mathrm{ml} / \mathrm{min}$. porti ad un aumento del $\mathrm{Kt} / \mathrm{V}$ da 0.82 a 1.18 . A seguito di questo incremento di dose erogata si osservò un miglioramento sia della morbilità che della mortalità di questi malati (Tab. II).

Un altro importante studio che dimostra l'importanza ineludibile della quantificazione del trattamento dialitico, ci viene offerto da T.F. Parker et al (5) riguardante il periodo 1989-1992 su 14.000 dialisi valutate con il sistema dell'Urea Kinetic Modeling (UKM), con il Kt/V e l'Urea Reduction Ratio (URR).

Da questa indagine si è osservato come un aumento da 195 a $220 \mathrm{~min}$. (+13\%) di durata della dialisi, determinasse un incremento della clearance dell'urea da 222 a $266 \mathrm{ml} / \mathrm{min}$. (+20\%) e comportasse una variazione di $\mathrm{Kt} / \mathrm{V}$ da 1.18 a $1.46(+24 \%)$; veniva così dimostrato che i maggiori effetti in termini di riduzione dell'urea si ottenevano aumentando $\mathrm{i} \mathrm{Kt} / \mathrm{V}$ da valori $<1$, a valori $>1$.

Non si avrebbero, invece, ulteriori evidenti vantaggi quando il $\mathrm{Kt} / \mathrm{V}$ è al di sopra di 1.50 .

La valutazione della mortalità in questa popolazione ha contemporaneamente dimostrato una sua riduzione inversamente proporzionale alla quantità di dialisi somministrata, passando dal $22.5 \%$ del 1989 all'inizio dello studio, al 18.1\% del 1992 alla fine dello studio.

Gli Autori concludevano che era possibile migliorare la sopravvivenza nella popolazione in dialisi negli USA con maggiore quantità di dose dialitica, stimando così un risparmio da 8.000 a 16.000 vite umane all'anno monitorando la cinetica dell'urea.

I vari studi citati concordano nel ritenere come l'adeguatezza dialitica monitorata con l'UKM rappresenti un indice indispensabile di valutazione per migliorare la sopravvivenza di questi pazienti.

L'UKM misurato nell'acqua plasmatica rappresenta a tutt'oggi il modello più accurato per lo studio della rimozione dell'urea. Questa tecnica non ci permette però di seguire gli scambi ionici tra i diversi compartimenti, in particolare in quello intracellulare.

\section{TABELLA II}

\begin{tabular}{lcccc}
\hline Anno & $\begin{array}{c}\text { PTS } \\
(\mathrm{n})\end{array}$ & $\begin{array}{c}\mathrm{t} \text { di dialisi } \\
\text { (minuti) }\end{array}$ & $\begin{array}{c}\text { Kt/V } \\
\text { (media) }\end{array}$ & $\begin{array}{c}\text { Morbilità } \\
\text { (gg. Osp/anno) }\end{array}$ \\
\hline 1988 & 92 & 195 & 0.82 & 15.2 \\
1991 & 130 & 212 & 1.18 & 10.3 \\
\hline
\end{tabular}


Questo comparto risulta tuttora un campo poco studiato nel corso della dialisi, per cui si ritiene che un sostanziale progresso alla conoscenza della fisiopatologia del paziente uremico, potrà essere conseguito solo mediante l'impiego di sensori in grado di monitorare oltre al "milieu intérieur", il metabolismo cellulare.

\section{BIBLIOGRAFIA}

1. Clark WR, et al. Quantification of hemodialysis: analysis of methods and the relevance to patient outcome. Blood Purf 1997; 15: $92-$ 111.

2. Gotch FA, Sargent JA. A mechanistic analysis of National Cooperative Dialysis Study (NCDS). Kidney Int 1985; 28: 526-34.

3. Bellco Marketing Dept. Tetta C, Santoro A. Cinetica dell'urea, 1996. Possibilità di valutazione "online" dell'ultrafiltrato. Tecniche Nefrologiche e dialitiche 1994; 6: 0000.

4. Hakim RM, et al. Effects of dose of dialysis on morbility and mortality. Am J Kidney Dis 1994; 23: 661-9.

5. Parker TF, et al. Survival of hemodialysis patients in the United States is improved with a greater quantity of dialysis. Am J Kidney Dis 1994; 23: 670-80. 